\title{
Influência do avanço cinemático das rodas dianteiras sobre a eficiência em tração de tratores com quatro rodas motrizes não isodiamétricas ${ }^{1}$
}

\author{
Influence of the kinematics advance on the traction efficiency of the front wheel assist tractor
}

\author{
José Fernando Schlosser ${ }^{2}$ Pilar Linares ${ }^{3}$ Luis Márquez ${ }^{3}$
}

\section{RESUMO}

O objetivo deste experimento foi estudar a influência da relação cinemática de interferência entre os dois eixos sobre a eficiência em tração dos tratores de tração em quatro rodas. Deste experimento, pode-se concluir que a maior eficiência em tração se obtém sempre com relações cinemáticas próximas a um, isto é, com as velocidades periféricas iguais das rodas. $\mathrm{Na}$ condição de solo firme, o trator absorve as vibrações decorrentes da interferência entre eixos $e$, no solo macio, este se romperá, facilitando o desenvolvimento da tração, sempre com índices de patinagem maiores nas rodas dianteiras.

Palavras-chave: influência cinemática, tratores, pneus.

\section{ABSTRACT}

The objective of this experiment was to study the effect of cinematic relationship between trator axles on traction efficiency of four-wheel tractor drive system. Results indicated that a higher traction efficiency was observed when cinematic relationship was close to one (using the same peripheral wheel speed). Considering a hard soil surface the trator absorbed vibrations due to interference between axles. However, on a soft soil surface the trator traction was smooth with a higher front wheel slippage index.

Key words: kinemactis influence, tractors, tires.

\section{INTRODUÇÃO}

Pela própria polivalência que rege o projeto do trator, a indústria incrementa os tamanhos das séries industriais, reduzindo os custos de produção industrial, obrigando o usuário a adaptar o trator às circunstâncias de cada trabalho agrícola, o que consegue de uma maneira especial, selecionando o tipo de pneus e suas pressões internas, assim como os pesos extras móveis para alcançar uma massa total e uma distribuição entre eixos adequada ao tipo de trabalho que realiza.

Neste aspecto, com a incorporação da tração dianteira auxiliar no eixo dianteiro, com rodas de menor tamanho, surgem complicações adicionais, já que, normalmente, entre os dois eixos do trator não se incorpora um diferencial que compense as diferenças cinemáticas que se produzem entre rodas dianteiras e traseiras, estabelecendo-se, por construção, uma discordância cinemática fixa, que é o resultado de um compromisso técnico para condições "médias" de campo, mas que quase nunca é a melhor solução para as circunstâncias reais de trabalho. O usuário tem condições de mudar a diferença cinemática de origem, conseqüência da velocidade de giro de cada roda e do raio dinâmico do pneu utilizado. Ao selastrar o trator para as diferentes operações de campo, modificando a pressão interna dos pneus e a posição relativa das massas de lastro, pode chegar à solução mais vantajosa para cada circunstância.

A dinâmica de tração depende principalmente da forma como se realiza a transmissão da potência desde o motor até os eixos, por meio de um diferencial entre eles ou por meio de um acoplamento rígido, como explica CHUDAKOV (1977). É imprescindível dizer que se a ligação entre os eixos é rígida, haverá obrigatoriamente uma relação invariável entre as velocidades angulares dos eixos. Nesta configuração, pode ocorrer uma discordância cinemática entre os eixos, fazendo com que a velocidade teórica entre rodas possa variar, ainda que as velocidades reais de avanço nos eixos dianteiro e traseiro devam ser iguais.

\footnotetext{
${ }^{1}$ Parte da tese de doutorado do primeiro autor, apresentada na Universidad Politécnica de Madrid, Espanha.

${ }^{2}$ Engenheiro Agrônomo, Doutor, Professor Titular, Departamento de Engenharia Rural, Centro de Ciências Rurais, Universidade Federal de Santa Maria, Campus Universitário Camobi, 97105-900, Santa Maria, RS. Fone: 0xx552208850, E-mail: schlosse@ccr.ufsm.br. Autor para correspondência.

${ }^{3}$ Engenheiro Agrônomo, Doutor, Professor Titular, Departamento de Ingeniería Rural, Universidad Politécnica de Madrid, Espanha.
} Recebido para publicação 30.12.03 Aprovado em 02.06.04 
Existem muitos problemas ainda não explicados devidos à relação fixa entre as velocidades dos eixos dianteiros e traseiros, principalmente nas manobras, o que costuma chamar-se de interferência ("transmision wind ups”). RENIUS, K.T. (1994) observa que um número crescente dos tratores fabricados principalmente no Japão (introduzido por Kubotta em 1986) dispõe de um dispositivo no eixo que é ativado de modo automático quando o eixo dianteiro começa a modificar-se para realizar uma manobra, com o objetivo de neutralizar esta interferência. No projeto do trator, os técnicos impõem uma relação constante entre os eixos, mas a velocidade das rodas pode variar em função de vários fatores, entre eles, a transferência de peso, o desgaste diferenciado dos pneus e a pressão interna.

Tentando demonstrar a influência da tração dianteira auxiliar dos tratores, BASHFORD (1985) mediu a força de tração, a resistência ao rolamento e a patinagem nas rodas motrizes de um trator de rodas, com tração dianteira conectada e desconectada, chegando à conclusão de que as perdas por rolamento são relativamente independentes do modo como se conduz o trator, (com a tração dianteira ligada ou não) e da distribuição do lastro. No entanto, as perdas por patinagem foram dependentes da tração dianteira e da maneira como se usa o lastro.

Em estudo comparativo do rendimento de um trator com tração dianteira auxiliar, BASHFORD et al. (1985), com a tração dianteira acionada ou não, obtiveram dados em duas condições de solo distintas, superfície asfaltada e solo argiloso lavrado. A massa se manteve constante e se empregaram três porcentagens de distribuição de peso entre os dois eixos. Para mudar a relação de transmissão no eixo dianteiro, usaram-se quatro dimensões de pneus dianteiros diferentes, com os seguintes valores para a relação cinemática: 0,97, 1,01, 1,07 e 1,13. Em solo agrícola, a $4 \mathrm{~km} / \mathrm{h}$, os autores encontraram que a tração bruta não diferia nos tratamentos com relações cinemáticas maiores de 1 e eram superiores ao tratamento com a relação cinemática de 0,97 ao redor de $10 \%$. Em termos de eficiência de tração, os valores máximos foram alcançados com a relação cinemática de 1,01 entre os valores de 30 e $35 \%$ do peso total no eixo dianteiro. Porcentagens de peso dianteiro superiores a este valor provocavam diminuição de eficiência nas relações de 1,01 e 1,07, enquanto que, com as demais relações cinemáticas $(0,97$ e 1,13), o ponto de mínima eficiência correspondia à faixa entre os 30 e 35\% do peso total no eixo dianteiro.
Para proporcionar o mesmo torque nos dois eixos de um trator TDA, podem ser necessários três diferenciais, ou seja, um diferencial em cada eixo e o terceiro entre os dois eixos. No entanto, esta configuração pode ocasionar a paralisação do trator se a aderência das rodas é pobre. Nestas condições, o terceiro diferencial deverá ser bloqueado para ser operado. Por isto, na prática, não é muito utilizado este terceiro diferencial pelos fabricantes. Quando a TDA é acionada, ocorre uma ligação rígida entre os eixos, ocorrendo uma relação constante entre as velocidades angulares dos eixos. Esta relação constante é projetada pelos técnicos e denominada de adiantamento cinemático (RACKHAM \& BLIGHT, 1985).

Em operações como as que se pratica na agricultura, há uma crescente aceitação de tratores de tração auxiliar, pois transmitem melhor a potência às rodas, já que todo seu peso pode ser utilizado para a tração. Segundo este autor, a eficiência máxima em tração somente pode ser conseguida, neste tipo de trator, se a relação entre o patinamento das rodas dianteiras e traseiras é igual a 1, ou seja, o patinamento das rodas dianteiras é igual ao das traseiras. Assim, em condições normais, somente se consegue obter a máxima eficiência, em relação ao patinamento, quando este é igual nas rodas dianteiras e traseiras. Esta condição é necessária para a obtenção da máxima eficiência com relação ao patinamento das rodas (WONG, 1978).

O objetivo deste trabalho foi avaliar a influência da relação cinemática que permita aproveitar eficientemente a potência em tração com tratores com tração dianteira auxiliar.

\section{MATERIAL E MÉTODOS}

Foi utilizado um trator de rodas marca John Deere, modelo 6400, com eixo dianteiro motriz e transmissão tipo PowrQuad. Os pneus foram Michelin 16.9R24 BIB x M18 T2 134 A8 131B nas rodas dianteiras e Firestone Radial 700 18.4R38 146 A8 143B nas traseiras. A distribuição do peso nos eixos variava em função do tratamento e foram determinados com um operador e o equipamento adicionado para as medições. A relação entre o número de voltas das rodas dianteiras e voltas das rodas traseiras (adiantamento cinemático) é de 1,3828. Como unidade de carga, arrastou-se um trator John Deere, modelo 2800. 
Utilizou-se uma instrumentação eletrônica para medir as variáveis, marca Dickey-John, modelo DJ-CMS 100. Este controlador de rendimento recebe a informação de diferentes sensores, como o que permite o registro da velocidade de giro das rodas e o da medida da velocidade de avanço do trator, utilizando um radar de baixa freqüência. Este equipamento se completava com um sensor de forças de tração com capacidade de 5000 daN. As magnitudes medidas foram enviadas a um condicionador de sinais de 16 canais, com conversor analógico/digital, entradas lógicas e contador de impulsos, um indicador digital e uma unidade central de processamento e aquisição de dados. Um computador portátil tinha a função de armazenar, em disco, os dados obtidos durante o ensaio para posterior análise. A gestão do sistema se realizava mediante um "software” AGRONOMO.EXE versão 1.0, desenvolvido para o ensaio de tratores a campo.

As provas de campo foram realizadas no Centro de Formação da Companhia John Deere Ibérica SA, nas proximidades da cidade de Toledo, Espanha.

A relação cinemática $(K v)$ foi calculada a partir dos raios estáticos das rodas e da relação entre as velocidades angulares de cada eixo do trator:

$$
K v=\frac{r d}{r t} \cdot a
$$

Em que: $\quad r d=$ raio estático dianteiro sob carga

$$
r t=\text { raio estático traseiro sob carga }
$$$$
\mathrm{a}=\text { adiantamento cinemático }
$$

A área total do experimento foi dividida em parcelas. Cada uma delas correspondia a uma repetição, das três que se faziam para cada tratamento. Cada uma das parcelas tinha 3 metros de largura e 70 metros de comprimento total, com 60 metros de comprimento útil e o restante para estabilização da velocidade. Durante o trabalho, sempre se bloqueava o diferencial do trator de ensaio e se trabalhava com a tração dianteira conectada.

Os tratamentos aplicados eram relações cinemáticas obtidas pela variação do raio estático, decorrente de diferenças na distribuição de peso e pressão interna dos pneus, conforme a tabela 1 . Utilizou-se um delineamento estatístico inteiramente casualizado com nove tratamentos.

Os tratamentos se repetiam para duas condições de solo, firme (Índice de cone médio a 15 $\mathrm{cm}$ de profundidade $=1234 \mathrm{kPa}$ ) e preparado (Índice de cone médio a $15 \mathrm{~cm}$ de profundidade $=162 \mathrm{kPa})$. A análise estatística dos dados se fez utilizando o pacote estatístico SAS (Statistical Analisys System), procedendo-se na análise da variância e teste de diferenças de médias.

Em cada repetição determinou-se a velocidade de deslocamento, a resistência ao rolamento, a patinagem das rodas, a força de tração na barra, a eficiência na resistência ao rolamento $\left(\eta_{k}\right)$, calculada desta forma:

$$
\eta_{k}=\frac{Q}{Q+R R}
$$

A eficiência na patinagem $\left(\eta_{d}\right)$, calculada como a média entre as eficiências dos dois eixos com respeito a este parâmetro. Assim:

$$
\begin{gathered}
\eta_{\delta d}=1-\delta_{d} ; \quad \eta_{\delta t}=1-\delta_{t} ; \\
\eta_{\delta}=\frac{\eta_{\delta d}+\eta_{\delta t}}{2}
\end{gathered}
$$

e Eficiência em tração $\left(\eta_{B T}\right)$ : calculada como :

$$
\eta_{\text {в }}=\eta_{t} \cdot \eta_{k} \cdot \eta_{\delta}
$$

Em que: $\quad Q=$ Força de tração na barra, em $\mathrm{kN}$

$R R=$ Resistência ao rolamento, em $\mathrm{kN}$

$\delta^{d}=$ Patinagem das rodas dianteiras, \%

$\delta t=$ Patinagem das rodas traseiras, \%

$\eta_{\delta d}=$ Eficiência em relação à patinagem das rodas dianteiras, índice

$\eta_{\delta t}=$ Eficiência em relação à patinagem das rodas traseiras, índice

$\eta t=$ Eficiência na transmissão

A eficiência na transmissão foi estimada em 87\% para este trator com câmbio hidrostático.

Uma outra forma de calcular a relação cinemática é na condição dinâmica sob tração, isto é, calculada a partir dos dados das provas com tração e com as condições que supõe cada tratamento.

Em função de que havia dados disponíveis de velocidade teórica das rodas, os mesmos que se utilizavam para o cálculo da patinagem, calcularam-se as relações cinemáticas, assim:

Tabela 1 - Relações cinemáticas estáticas relacionadas com os tratamentos utilizados.

\begin{tabular}{ll}
\hline Tratamento & Relação cinemática \\
\hline 1 & 0,975 \\
2 & 0,977 \\
3 & 1,006 \\
4 & 1,028 \\
5 & 1,044 \\
6 & 1,051 \\
7 & 1,069 \\
8 & 1,085 \\
9 & 1,088 \\
\hline
\end{tabular}




$$
K_{v}=\frac{v_{d}}{v_{t}}
$$

Emque: $\quad V d=$ velocidade periférica da roda dianteira, $\mathrm{m} / \mathrm{s}$

$V t=$ velocidade periférica da roda

traseira, $\mathrm{m} / \mathrm{s}$

E se expressam como diferenças em relação à unidade, que é o valor que representa a igualdade de velocidades dos eixos.

$$
K_{v}(\%)=\left(1-K_{v}\right) \cdot 100
$$

\section{RESULTADOS E DISCUSSÃO}

A relação cinemática entre eixos do trator depende de dois fatores, primeiro da relação de velocidade entre os eixos e segundo dos raios das rodas dianteira e traseira do trator. O primeiro fator é fixo e depende do projeto do fabricante que o estabelece por meio das relações de transmissão entre os dois diferenciais. Geralmente esta relação de velocidades é maior que 1 , a favor do eixo dianteiro, que gira mais rápido que o traseiro. No trator estudado, a relação entre velocidades dos eixos faz com que o eixo dianteiro gire $38 \%$ mais rápido que o traseiro, para compensar a diferença de tamanho entre as rodas. O segundo fator é o próprio raio das rodas, que pode ser alterado pelo usuário, por meio da troca dos pneus ou pela variação da pressão interna dos mesmos.

Neste trabalho, conseguiram-se diferentes relações cinemáticas estáticas de 0,975 até 1,088 (Tabela 1), isto é, -2,25\% e 8,84\%, respectivamente. Com as primeiras, a relação cinemática é de atraso, as rodas traseiras giram tangencialmente mais rápido que as dianteiras e, no segundo caso, é de adiantamento, e as rodas dianteiras giram tangencialmente mais rápido que as traseiras. Conseguiu-se um escalonamento de relações estáticas de - 2,25\%; -2,45\%; 0,61\%; 2,84\%; 4,36\%; 5,06\%; $6,90 \% ; 8,50 \%$; 8,84\%, sendo duas de atraso (com sinal negativo), uma de neutralidade e seis de adiantamento cinemático estático. A condição estática supõe que a determinação se realize, utilizando o raio estático sob carga, com o trator parado sobre una superfície plana e regular, com as características de pressão interna e distribuição do tratamento a que se está fazendo referência.

Os raios estáticos sob carga aumentaram tanto no eixo dianteiro como no traseiro quando se diminuiu o peso sobre este eixo. O raio estático diminuía a medida que se utilizava uma pressão interna menor no pneu. Mesmo assim foi mais clara a influência da pressão interna do que a da distribuição de peso sobre os eixos.

Da análise estatística da relação cinemática, na condição dinâmica sob tração, na condição de solo firme, concluiu-se que a média geral foi de 1,013, o que indica um adiantamento de velocidade do eixo dianteiro em relação ao traseiro de $1,32 \%$, com um coeficiente de variação bastante baixo (2,93\%), o que indica boa precisão na tomada de dados (Tabela 2).

Pode-se afirmar que da comparação entre as diferentes relações cinemáticas é que a estática somente serve como referência de projeto e pode determinar quais são os raios dos pneus e as relações entre velocidades das rodas permitidas ao fabricante, ao conceber os parâmetros de projeto dos diferenciais, e as combinações de pneus permitidos para cada modelo de trator em função de suas características e da sua previsível condição dinâmica de tração e distribuição de peso. Desta relação, podese deduzir quais são os limites que o fabricante e o usuário podem aceitar para equipar e utilizar o trator. A relação estática baseada em raios sem carga, raios estáticos sob carga, raios índice informados por fabricantes, etc tem muita utilidade também para que se desenvolvam estudos teóricos, como fizeram alguns autores e que serviram de base para os trabalhos práticos que os seguiram.

A condição dinâmica em trabalho de tração, da relação cinemática entre os eixos, é a que realmente se pratica na realidade, pois os eixos realmente sofrem o efeito da tração, a resistência ao rolamento e por isto patinam. Na condição dinâmica, independente do estado de superfície, são os pneus, que absorvem as diferenças

Tabela 2 - Relações cinemáticas das duas condições de solo.

\begin{tabular}{lcccc}
\hline Tratamento & $\begin{array}{c}\text { Relação } \\
\text { cinemática } \\
\text { em solo } \\
\text { firme }\end{array}$ & $\begin{array}{c}\text { Diferença } \\
\text { percentual } \\
(\%)\end{array}$ & $\begin{array}{c}\text { Relação } \\
\text { cinemática } \\
\text { em solo } \\
\text { preparado }\end{array}$ & $\begin{array}{c}\text { Diferença } \\
\text { percentual } \\
(\%)\end{array}$ \\
\hline 1 & $1,011^{\mathrm{b}}$ & 1,11 & $1,048^{\mathrm{d}}$ & 4,81 \\
2 & $1,018^{\mathrm{c}}$ & 1,78 & $1,019^{\mathrm{c}}$ & 1,86 \\
3 & $0,999^{\mathrm{a}}$ & $-0,11$ & $1,062^{\mathrm{e}}$ & 6,17 \\
4 & $1,018^{\mathrm{c}}$ & 1,78 & $1,048^{\mathrm{d}}$ & 4,78 \\
5 & $1,031^{\mathrm{d}}$ & 3,11 & $1,024^{\mathrm{c}}$ & 2,43 \\
6 & $1,017^{\mathrm{c}}$ & 1,67 & $1,005^{\mathrm{b}}$ & 0,47 \\
7 & $0,996^{\mathrm{a}}$ & $-0,44$ & $0,991^{\mathrm{a}}$ & $-0,89$ \\
8 & $1,010^{\mathrm{b}}$ & 1,00 & $1,023^{\mathrm{c}}$ & 2,33 \\
9 & $1,019^{\mathrm{c}}$ & 1,89 & $1,010^{\mathrm{b}}$ & 1,02 \\
\hline
\end{tabular}

Médias com a mesma letra em índice não são significativamente diferentes a $95 \%$. 
entre as velocidades teóricas dos eixos em que estão colocados e têm que se deformar e elevarse, provocando com isto as freqüentes vibrações, que se notam no trator. Se a superfície do solo é dura, como em um caminho ou em solo firme seco, os pneus se desgastarão, suas garras se romperão e o eixo que gira mais rápido terá que encurtar a sua circunferência, enquanto o outro, que gira mais devagar o fará ao contrário. Em solo macio, como a condição de solo úmido ou fofo, comparável à situação de solo solto, é o solo que fará o papel de fusível, permitindo a maior patinagem do eixo que gira mais rápido. Como a condição é dinâmica, o efeito da tração fará que se transfira maior parcela do peso do eixo dianteiro ao traseiro, fazendo que este tenha maior coeficiente dinâmico de tração, portanto, recebendo maiores condições de eficiência em tração, o que nem sempre se manifesta em menores patinagens das rodas.

A eficiência na resistência ao rolamento média foi de $84 \%$ em solo firme e $77 \%$ em solo preparado. A eficiência, em relação à patinagem, foi de $89 \%$ para o solo firme e $76 \%$ para a condição de solo preparado. A eficiência na patinagem dos pneus dianteiros média foi de $88 \%$ para solo firme e $76 \%$ para solo preparado. Os maiores valores estavam relacionados ao tratamento em que o avanço cinemático era maior $(8,8 \%)$ e que se reduzia a zero na condição dinâmica, em solo firme. Em solo preparado, a mesma tendência se repetiu.

A eficiência em tração média foi bastante menor em solo preparado (51\%) comparada ao solo firme (66\%). Para o solo firme, as menores eficiências foram obtidas no tratamento de menor relação cinemática, isto é, de atraso, e a maior eficiência foi obtida no tratamento de maior relação cinemática, com $8,8 \%$ de adiantamento, embora o tratamento 2 não tenha diferido estatisticamente do mais eficiente e o tratamento 6 não tenha diferido estatisticamente do menos eficiente. Em solo preparado, foi estatisticamente comprovado que os tratamentos com maiores relações cinemáticas apresentassem maiores eficiências, e os com menores relações cinemáticas apresentassem menores eficiência em tração (Tabela 3), dentro dos limites estudados.

\section{CONCLUSÕES}

Não houve qualquer relação entre as relações cinemáticas calculadas a partir dos raios estáticos e as relações cinemáticas calculadas a partir das velocidades das rodas em tração, nas duas
Tabela 3 - Eficiência em tração (\%), em experimento de tração em duas condições de solo.

\begin{tabular}{lcc}
\hline Tratamento & $\begin{array}{l}\text { Eficiência em } \\
\text { tração em solo } \\
\text { firme }\end{array}$ & $\begin{array}{l}\text { Eficiência em tração } \\
\text { em solo preparado }\end{array}$ \\
\hline 1 & $0,63^{\mathrm{a}}$ & $0,58^{\mathrm{bc}}$ \\
2 & $0,69^{\mathrm{c}}$ & $0,55^{\mathrm{a}}$ \\
3 & $0,66^{\mathrm{b}}$ & $0,55^{\mathrm{a}}$ \\
4 & $0,66^{\mathrm{b}}$ & $0,57^{\mathrm{b}}$ \\
5 & $0,66^{\mathrm{b}}$ & $0,58^{\mathrm{bc}}$ \\
6 & $0,61^{\mathrm{a}}$ & $0,54^{\mathrm{a}}$ \\
7 & $0,66^{\mathrm{b}}$ & $0,58^{\mathrm{bc}}$ \\
8 & $0,66^{\mathrm{b}}$ & $0,59^{\mathrm{bc}}$ \\
9 & $0,69^{\mathrm{c}}$ & $0,60^{\mathrm{c}}$ \\
\hline
\end{tabular}

Médias com a mesma letra em índice não são significativamente diferentes a $95 \%$.

condições de solo estudadas. Na condição de solo duro, o tratamento que produziu a menor patinagem nas rodas traseiras coincidiu com a menor relação cinemática dinâmica. Na condição de solo macio, as menores patinagens ocorreram nas condições dos tratamentos com menor porcentagem de peso nas rodas dianteiras e maiores raios estáticos nestes pneus, isto é, maiores valores da relação cinemática. Com respeito à relação cinemática, para cada condição de solo, calculada a partir das velocidades teóricas das rodas, constatou-se que houve uma diminuição importante da eficiência em tração com o aumento desta. Em solo firme, a diminuição é maior que em solo preparado. A relação cinemática calculada, utilizando-se os raios estáticos, pode ser utilizada como referência para o projeto e a fixação da dimensão dos pneus.

\section{REFERÊNCIAS BIBLIOGRÁFÍCAS}

BASHFORD, L.L. Axle power distribuition for a front-wheel assist tractor. Transactions of the ASAE, v.28, n.5, p.13851388, 1985.

BASHFORD, L.L.; WOERMAN, G.R.; SHROPSHIRE, G.J. Front wheel assist performance in two and four-wheel drive modes. Transactions of the ASAE, v.28, n.1, p.2329, 1985 .

CHUDAKOV, D.A. Fundamentos de la teoría y el cálculo de tratores y automóviles. Moscou : Mir Moscú, 1977. 435p.

RACKHAM, D.H.; BLIGHT, D.P. Four wheel drive tractors A review. Journal of Agricultural Engineers, v.56, p.185201, 1985.

RENIUS, K.T. Trends in tractor design with particular reference to Europa, Journal of Agricultural Engineers, v.57, p.322, 1994

WONG, J.Y. Theory of ground vehicles. New York : John Wiley \& Sons, 1878. 330p. 